DRUG UTILIZATION PATTERN FOR OCULAR ALLERGIES IN A TERTIARY CARE TEACHING HOSPITAL OF ASSAM

\title{
Medicine
}

\section{Luna Kuli*}

Post Graduate student, Assam Medical College and Hospital, Dibrugarh, Assam *Corresponding Author

Nilotpal Barua Associate Professor, Assam Medical College and Hospital, Dibrugarh, Assam,

\section{ABSTRACT}

Introduction: The importance of conducting drug utilization pattern studies is to study the prescribing trends to promote rational prescribing \& develop awareness amongst prescribers. Aim and Objectives: To evaluate the current prescribing and drug utilization pattern in the treatment of ocular allergies in a tertiary care teaching hospital of Assam. Materials and Methods: A total of 265 prescriptions were assessed. A retrospective study was conducted at the Department of Ophthalmology and Pharmacology, Assam Medical College and Hospital, Dibrugarh, Assam for a period of 2 months from 1/01/2018 to 28/02/2018. Patients of either sex and all ages were included. Patients with concomitant diseases like diabetes, hypertension or other diseases were excluded.Results: The commonest ocular allergy was simple allergic conjunctivitis. The male: female ratio was 1.95 . Commonest age group affected by the disease is 31-40 years. The average number of drugs per prescription was 1.68 . Olopatadine was the most commonly prescribed drug. Topical route was the commonest. The demographic details of the patient such as name, age, sex, address, chief complaints, findings, diagnosis, Rx symbol, doctor's signature were present in all prescriptions. Conclusion: No polypharmacy or irrational drug use were noticed.

\section{KEYWORDS}

- drug utilization study, ocular allergies, tertiary care hospital of Assam

\section{INTRODUCTION}

Drug utilization studies has been described by the World Health Organization (WHO) as the marketing distribution, prescription and use of drugs in a society, with special emphasis on the resulting medical, social and economic consequences.

It is an important aspect of pharmacoepidemiology which explains the degree, nature and risk factors of drug exposure with the final objective of rational use of drugs in the community.

It adds to rational use of drugs by exploring the trends in drug use, producing early signs of irrational drug use and proposes interventions to refine drug usage.3Prescription pattern studies are studies aimed at analysing the prescribing, dispensing and administration of drugs in a clinical set up to encourage rational prescribing amongst prescribers. 4 The importance of conducting drug utilization pattern studies is to study the prescribing trends to promote rational prescribing \& develop awareness amongst prescribers.

Formerly, only a few studies were carried out to evaluate drug use trends for ocular allergies in ophthalmology in India. Hence, the present study was carried out to evaluate the drug use pattern in ocular allergies in Ophthalmology department in a tertiary care teaching hospital of Assam.

Ocular allergies are a group of external ocular conditions resulting in inflammatory reaction of the ocular surface from one or more types of hypersensitivity reactions to allergens. It is a widespread issue that affects people of all ages in which the manifestation may vary from being milder asymptomatic cases to critical vision threatening inflammation cases. 6 Patients affected with ocular allergies manifests with crucial exhausting symptoms like itching, watering of eyes and ropy discharge which impact their routine work, rendering problems in focusing in their work, thereby influencing their quality of life.7 The causative agents include pollen dust, household dust, mould, spores, animal exfoliations or chemical medium etc. ${ }^{8}$

The disease spectrum is categorized under the following five subtypes: ${ }^{9,10}$

- Seasonal Conjunctivitis

- Vernal Conjunctivitis

- Atopic Conjunctivitis

- Giant Papillary Conjunctivitis

- Contact Hypersensitivity

Innumerable case reports suggest that the incidence and prevalence of ocular allergies have grown substantially around the world during the last 40 years and they continue to increase.
It would be informative, to evaluate the trend of anti- allergic drug use in the current system as drug utilization studies related to ocular allergies from India are scanty. With this viewpoint, this study was undertaken to determine the drug utilization pattern for ocular allergies in a tertiary care teaching hospital of Assam.

The prescription pattern study in patients with ocular allergies patients in Assam, hence has been outlined to provide an ethical feedback to the prescriber.

This study evaluates the prescribing pattern as per the WHO drug indicators, the number of drugs per prescription, the disease statistics as per age and sex, the commonly prescribed drugs and the percentage of different drug groups, the percentage of different drug formulation used, fixed dose combinations, usage of brand names, and drugs prescribed from National List of Essential Medicines 201511 and Essential Drug List of Assam 2016

\section{AIMS AND OBJECTIVES:}

To evaluate the current prescribing and drug utilization pattern in the treatment of ocular allergies in a tertiary care teaching hospital of Assam and to assess the rationality of drug use.

\section{MATERIALS AND METHODS:}

Requisite permission for conducting the study was obtained from the institutional authority before performing the study.

Study site: This study was conducted at the Department of Ophthalmology and Department of Pharmacology, Assam Medical College and Hospital, Dibrugarh, Assam.

Study period: A retrospective study was carried out for a period of two months from $1 / 01 / 2018$ to $28 / 02 / 2018$

Study design: Retrospective study.

Sample size: A total of 265 prescriptions were evaluated.

Inclusion criteria: Prescriptions of diagnosed patients of ocular allergies of either sex and all ages were included.

Exclusion criteria: Prescription of patients with concomitant diseases like diabetes, hypertension or other diseases were excluded.

\section{RESULTS AND OBSERVATION:}

A total of 265 prescriptions were evaluated for the drugs used for ocular allergies in patients in Ophthalmology department in a tertiary care teaching hospital in Assam. 
Total number of drugs prescribed were 445.

The average number of drugs per Drugs per prescription was 1.679.

Out of all different types of ocular allergies, Simple Allergic Conjunctivitis was $147(55.47 \%)$, vernal keratoconjunctivitis was $38(14.33 \%)$, atopic keratoconjunctivitis was $35(13.20 \%)$, giant papillary conjunctivitis was $27 \quad(10.18 \%)$ and phlyctenular conjunctivitis was $18(6.79 \%)$

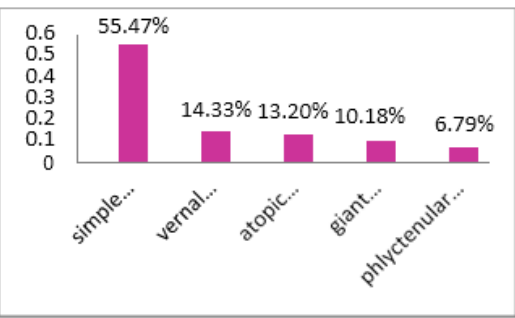

The male : female ratio was $1.944 .$, Male $=175(66.03 \%)$, Female $=$ $(33.96 \%)$ cases were seen.

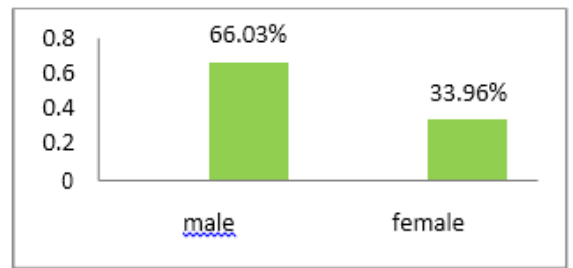

Age-wise statistics(\%) of patients was 1-10 yrs was $30(11.29 \%)$, 1120 yrs was 43 (16.12\%), 21-30 yrs was 56 (20.96\%), 31-40 yrs was 64 $(24.19 \%), 41-50$ yrs was $34(12.90 \%), 51-60 y r s$ was $17(6.45 \%), 61$ 70 yrs was $21(8.06 \%)$.

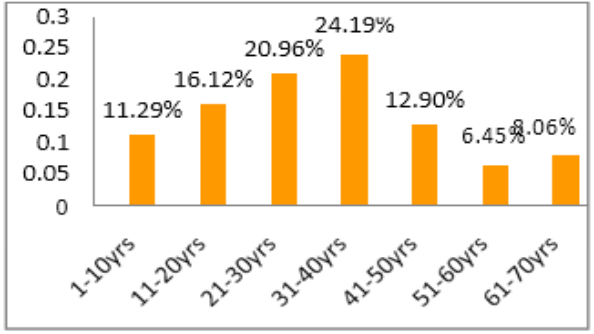

Single drug was prescribed in $81(18.16 \%), 2$ drugs were prescribed in $357(80.21 \%)$, and 3 drugs were prescribed in $7(1.57 \%)$.

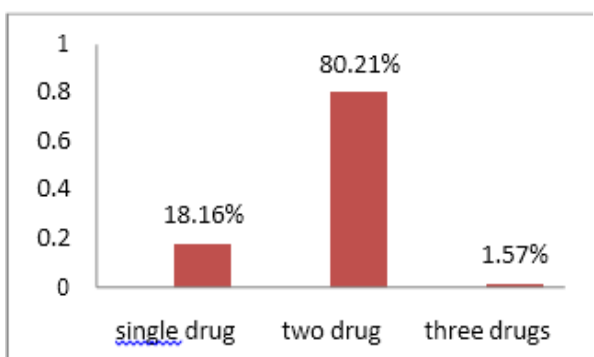

Antihistamine eye drops were most commonly prescribed 188 (42.28\%) followed by lubricating eye drops 161 (36.24\%) and antimicrobials $69(15.43 \%)$ and steroids $27(6.04 \%)$

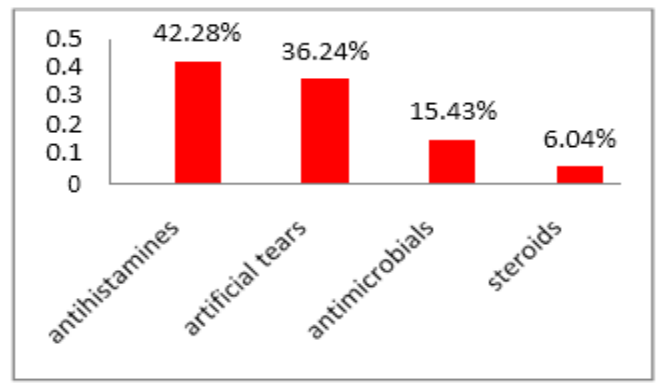

Topical $442(99.32 \%)$, oral 3(0.67\%), injectable zero.

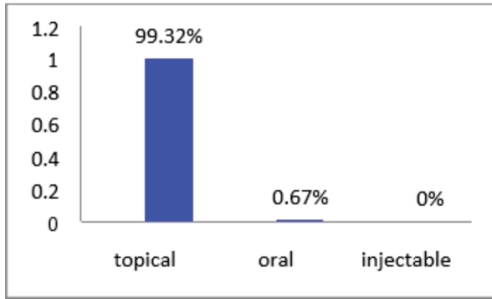

DISCUSSION:

Drug utilization study is a significant facet of pharmacoepidemiology which depicts the degree, nature and risk factors of drug exposure with the final goal of rational drug use in the community.

In India, an estimated $20 \%$ of the general population are affected from some form of ocular allergies presently.

Hence, it would be informative, to evaluate the trend of anti-allergic drug use in the current system as drug utilization studies related to ocular allergies from India are scanty.

With this viewpoint, this study was undertaken to determine the drug utilization pattern for ocular allergies in a tertiary care teaching hospital of Assam

So, the Present study analyzed prescriptions of 265 patients of Ophthalmology Out-Patients who were diagnosed with Ocular Allergies. Simple Allergic Conjunctivitis was the most common type of ocular allergy. The most commonly affected age group is $31-40$ yrs $(24.19 \%)$. The male : female ratio was 1.944 i.e. Male $175(66.03 \%)$ patients was more as compared to Female (33.96\%). Total 445 drugs were prescribed and the average number of drugs per prescription was 1.679. Most drugs were given by topical route of drug administration. Olopatadine was the common antihistaminic prescribed. Carboxymethyl Cellulose was prescribed commonly as artificial tear. The Antimicrobial most commonly prescribed was Moxifloxacin. 165 drugs were prescribed from the Essential Drug List of Assam 2016 (EDL Assam 2016) and 222 (49.66\%) were prescribed from National List of Essential Medicines 2015 (NLEM 2015) and zero brand names were used in the prescriptions.

Polypharmacy was found in zero prescriptions. The demographic details of the patient such as name, age, sex, address, chief complaints, findings, diagnosis, Rx symbol, doctor's signature were present in all prescriptions.

Antimicrobial therapy was given only to some patients to reduce the incidence of complications in future. Steroids were given for rapid cure. Most patients were treated with only antihistaminics or artificial tears and other supportive agents which is encourages good practice and reduce the cost of drug also for the patient.

The assessement for the cost of drug, duration of therapy was not done, as the study displayed quantitative data of drug usage pattern of ocular allergies in patients.

Hence, there is lot of scope to conduct similar studies in the future. The present study will help provide right feedback to the prescriber.

\section{ACKNOWLEDGEMENT:}

I would like to thank Dr. Jawahar Jyoti Kuli, Professor and Head of Department of Ophthalmology and Dr. Jayant Ekka (Post graduate) from the Department of Ophthalmology, Assam Medical College, Dibrugarh for permitting me to perform the study and for the provision of requisite data for my study.

\section{CONCLUSION:}

The Present study showed that no polypharmacy or irrational drug use was noticed. It is hence necessary to carry forward such type of similar studies periodically to assess the rational prescribing for treatment of ocular allergies.

\section{REFERENCES:}

1. Lee D and Bergman U, Studies of drug utilization in pharmacoepidemiology (4thedition). Storm LB (editor) John Wiley \& Sons Ltd England, 2005;401-417. Slathia A, Gupta V, Nanda R, Mahajan P. Drug UtilizationPatterninOphthalmology:A Observational and Cross-sectional Study. Int J Sci Stud 2017:5(5):63-65. 
3. Rajesh Kumar Suman ,Y A Deshmukh,Ipseeta Ray Mohanty,Varshav S. Gore. Drug Utilization studies in Glaucoma patients at MGM medical College and Hospital. International Journal Of Scientific Research. July 2013.volume:2, Issue: 7: 433-435.

4. Suman RK, Gore VS, Mohanty IR et. al. prescribing patterns of drugs used for treatment of conjunctivitis in ophthalmology outpatient department of tertiary care hospital. Int J Health Sci Res. 2015; 5(3):194-19.

5. Palain S, Shankar PR, Hegde C, Hegde M, Ojha P, and Mishra P. Drug utilization pattern in dental out patients in Tertiary care teaching hospital in western Nepal NYSDJ, 2008; 63-67.

6. Kubaisi B, Samra KA, Syeda S, et al. Ocular Allergy: an Updated Review. J Allergy Immunol 2017; 1:002

7. Kahol, P., Clinical Epidemiology and Global Health(2018), https:// doi. org/10.1016/j. cegh. 2018.07.001

8. Stock EL. Pendleton RB, Pharmacological treatment of Ocular allergic disease. Intl.Ophthalmol Clinics. 1993;33:47

9. Allansmith MB, Korm DR, Greiner JV et al. Giant Papillary Conjunctivitis in Contact lens wearer. Am J Ophthalmol. 1997;83:697.

10. Greoner JV, Convington HL, Allansmith MR. Surface morphology of giant papillary Conjunctivitis in contrast to normal contact lens wearer. Am J Ophthalmol.1975;85:242.

11. National List of Essential Medicines (NLEM) 2015. Available at: http:// cdsco. nic. in/ Write ReadData/NLEM-2015/NLEM,\%202015.pdf

12. Essential Drug List 2016, Health \& Family Welfare Department, Government of Assam. Available at: https://nhm.assam.gov.in/sites/default/files/swf_u tility folder/ departments/nhm_lipl_in_oid_6/do_u_want_2_know/1-PST_EDL.pdf 